\title{
Wenn Ausbildungsbetriebe Geschlecht auswählen. \\ Geschlechtsspezifische Lehrlingsselektion am Beispiel des Autogewerbes
}

\author{
Christian Imdorf
}

Published as:

Imdorf, Christian (2012). Wenn Ausbildungsbetriebe Geschlecht auswählen.Geschlechtsspezifische Lehrlingsselektion am Beispiel des Autogewerbes. In: M.M. Bergman, S. Hupka-Brunner, T. Meyer \& R. Samuel (Hrsg.), Bildung - Arbeit - Erwachsenwerden. Ein interdisziplinärer Blick auf die Transition im Jugend und jungen Erwachsenenalter. Wiesbaden: VS-Verlag für Sozialwissenschaften, 243-264.

DOI: $10.1007 / 978-3-531-19071-6 \_12$

Original source of publication: http://link.springer.com/chapter/10.1007\%2F978-3-531-19071-6_12

The final publication is available at www.springerlink.com

\section{Einleitung}

Frauen und Männer arbeiten auf dem Schweizer Arbeitsmarkt in unterschiedlichen Berufen - zum Nachteil der Frauen. Dem geschlechtersegregierten Arbeitsmarkt ist ein vergleichbar geschlechtersegregiertes Berufsausbildungssystem vorgelagert (Leemann und Keck 2005), welches von zwei Dritteln der Jugendlichen durchlaufen wird. Die Ausbildung findet zu über $80 \%$ in Betrieb und Berufsschule statt, wobei rund 230 meist geschlechtertypische Lehrberufe zur Wahl stehen (BBT 2011). Dass Jugendliche mehrheitlich geschlechtertypische Ausbildungsberufe erlernen, ist gut belegt (Borkowsky 2000; Leemann und Keck 2005: 121ff; Meyer et al. 2003: 25-27; SKBF 2010: 157f). Die Geschlechtersegregation des Ausbildungsmarkts überträgt sich durch die berufsförmige Kopplung von Ausbildungs- und Berufsstruktur nahezu bruchlos auf das Beschäftigungssystem (Trappe 2006: 52).

Wie aber kommt es zur frühen Vergeschlechtlichung der Ausbildungslaufbahnen? Auf dem Lehrstellenmarkt werden die Ausbildungsstellenangebote der Betriebe mit der Nachfrage der Jugendlichen marktförmig abgeglichen. Die Geschlechtersegregation des Ausbildungsmarkts kann somit das Resultat des Berufswahlverhaltens der Lehrstellensuchenden sowie des betrieblichen Einstellungsverhaltens sein. Für das Verständnis der geschlechterdifferenzierten Berufsfindung am Ende der obligatorischen Schulzeit erweisen sich subjekttheoretische Erklärungsansätze als besonders plausibel, welche die 
biographische Geschlechterkonstruktion von adoleszenten Jugendlichen durch ihre Identifizierung mit geschlechtertypischen Berufsbildern hervorheben (Brandt und Cornelißen 2004). Vergeschlechtlichte Berufsrollen erleichtern den Jungen und Mädchen entsprechende Männlichkeits- bzw. Weiblichkeitskonstruktionen (Gianettoni et al. 2010).

Es käme allerdings einer unzulässigen Verkürzung gleich, die Geschlechtersegregation des Ausbildungsmarkts ausschließlich den beruflichen Orientierungs- und Identitätsfindungsprozessen Jugendlicher zuzuschreiben. Sobald die Arbeitgeber Jugendliche bei der Ausbildungsplatzvergabe aufgrund der Geschlechterzugehörigkeit nicht gleichwertig beurteilen, tragen auch Erstere zur Geschlechtersegregation des Ausbildungssystems bei. Die internationale Forschung hat dabei verschiedentlich Hinweise ergeben, dass Ausbildungsbetriebe die Kanalisierung junger Männer und Frauen in geschlechtertypische Berufslaufbahnen mitverantworten. Gemäß Rauch und Schober (1996) wird die berufliche Geschlechtersegregation durch das Einstellungsverhalten von Betrieben gestützt. Auch bei Mariak und Kluge (1998: 209ff) finden sich arbeitgeberseitige Belege, wonach in männertypischen Branchen (Metallbau) männliche Auszubildende und in frauentypischen Branchen (Verkauf) weibliche Auszubildende präferiert werden (vgl. für Großbritannien Fuller et al. 2005: 304f.). Auch geschlechtsspezifische Nachfragedaten einer Erhebung des Bundesinstituts für Berufsbildung belegen, dass junge Frauen insbesondere dann größere Zugangsprobleme in den Ausbildungsmarkt haben, wenn sie auf der Suche nach einem Ausbildungsplatz in einem männertypischen Beruf waren (BMBF 2003: 44). Dabei scheinen insbesondere kleinere und mittlere Ausbildungsbetriebe (und weniger die Großbetriebe) Geschlecht als Auswahlkriterium bei der Vergabe von Ausbildungsplätzen durchzusetzen (Mariak und Kluge 1998: 210; Miller et al. 2005: 60).

Für die Schweiz mangelt es bisher an Studien, welche die Rolle der Ausbildungsbetriebe bei der Reproduktion der Geschlechtersegregation auf dem Ausbildungsmarkt untersucht haben. Der vorliegende Beitrag setzt bei dieser Forschungslücke an und analysiert exemplarisch am Beispiel des Autoreparatur- und Karosseriegewerbes die Bedeutung des Geschlechts bei der Auswahl von Auszubildenden. Diese Branche bietet sich an, da es sich bei den untersuchten 
Ausbildungsberufen - Automonteur/in, Automechaniker/in und Autolackierer/in ${ }^{1}$ zum einen um berufsbildungsstatistisch häufig erlernte Berufe handelt, die typischerweise in Klein- und Mittelbetrieben ausgebildet werden. Zum anderen betrug der Frauenanteil in diesen Berufen im Jahr 2005 nur 6,3\% ${ }^{2}$, womit es sich um eine ausgeprägt männertypische Branche handelt.

Ziel dieses Beitrags ist es, die Bedeutung des Geschlechts bei der Vergabe von Ausbildungsplätzen aus betrieblicher Perspektive theoretisch und empirisch zu klären. Aufgrund welcher betrieblichen Motive wird das Bewerbermerkmal Geschlecht bei der Auswahl von Auszubildenden bedeutsam? Inwiefern fördert die Ausbildungsplatzvergabe des Auto- und Karosseriegewerbes die Geschlechtstypik dieser Branche?

\section{Ein konventionensoziologisches Modell der Ausbildungsplatzvergabe}

Die Soziologie der Konventionen ermöglicht eine Konzeptualisierung der betrieblichen Auswahl von Auszubildenden (Imdorf 2010a, 2012; Imdorf und Leemann 2012). Sie gibt Antworten auf die Frage, wie Personalverantwortliche bei der Auswahl von Auszubildenden unter unsicheren Entscheidungsbedingungen möglichst konfliktfreie Ausbildungsverhältnisse antizipieren können. Die Problemlösung besteht in der Vorwegnahme der individuellen Verhaltensabstimmung, indem künftige Ausbildungs- und Arbeitssituationen mit Hilfe von Konventionen interpretiert und dabei u.a. die Verhaltenserwartungen der Belegschaft antizipiert werden. Konventionen sind Koordinationsprinzipien, die Arbeits- bzw. Ausbildungsorganisationen zusammenhalten. Sie sind als Reaktion auf Unsicherheit in solchen Organisationen entstanden und gewährleisten deren Bestandserhalt. Auf der Grundlage solcher Konventionen beurteilen die Personalverantwortlichen bei der Ausbildungsplatzvergabe die zukünftige, möglichst friktionsfreie Passung einer neuen auszubildenden Person in ein bestehendes Kollektiv von Mitarbeitenden und in den Betrieb als Ganzes (ausführlicher zum Konventionenbegriff vgl. Imdorf 2012).

\footnotetext{
${ }^{1}$ Es handelt sich hier um die offiziellen Schweizer Bezeichnungen der Ausbildungsberufe des Autound Karosseriegewerbes zum Zeitpunkt der Datenerhebung im Jahr 2005. Inzwischen wurden die Berufe modernisiert und umbenannt.

2 Eigene Berechnungen auf Basis der Berufsbildungsstatistik (BFS 2006). Unter den Ausbildungsberufen des Autogewerbes sticht das Lackierereigewerbe mit einem für männertypische Berufe relativ hohen Frauenanteil von 17,9\% hervor. Der Frauenanteil in den Ausbildungsberufen Automonteur/in und -mechaniker/in betrug 2005 dagegen nur 4,2\%.
} 
In ihrer Rechtfertigungstheorie haben Boltanski und Thévenot (2007) eine Vielfalt von Konventionen des sozialen Zusammenhalts in unterschiedlichen ,Welten " vorgeschlagen: Diese Welten - sie heißen u.a. häusliche, staatsbürgerliche, industrielle oder marktförmige Welt - zeichnen sich durch ihr zentrales Koordinations- und Ordnungsprinzip aus: Tradition, Solidarität, Effizienz bzw. Konkurrenz. Sie unterscheiden sich darin, welche Qualitäten von Personen als besonders wertvoll erachtet werden, damit der soziale (bzw. hier: der betriebliche) Zusammenhalt zum Wohle aller garantiert oder zumindest antizipiert werden kann.

Ausbildungsbetriebe können nun als situative Kontexte verstanden werden, in denen die Koordinationsprinzipien dieser Welten und die daraus resultierenden Anforderungen an die berufliche, arbeitsförmige und soziale Passung konkurrieren und in der Personalselektion aufeinander abgestimmt werden müssen. Da kein Bewerber die Anforderungen eines Betriebs bezüglich all dieser Welten annähernd perfekt erfüllen kann, brechen Personalverantwortliche ihre Suchbemühungen ab, sobald sie eine Kandidatin gefunden haben, welche die multiplen Anforderungen genügend befriedigt. Es wird dann zu Gunsten einer/eines Kandidaten/in entschieden, von der/dem erwartet werden kann, dass sie/er dem Betrieb keine namhaften Probleme verursachen wird.

Um bei der Auswahl von Auszubildenden den Entscheidungsprozess zu beschleunigen und gleichzeitig das künftige Problemrisiko gering zu halten, greifen die Ausbildungsverantwortlichen auch auf askriptive Kategorien zurück, die störungsfreie Ausbildungs- und Arbeitsprozesse versprechen (zur Bedeutung der Nationalität sowie des Alters bei der Auswahl von Auszubildenden vgl. Imdorf 2010a, 2012). Auch die bereits aus den Vornamen in den Bewerbungsunterlagen interpretierbare Geschlechterzugehörigkeit kann den Selektionsverantwortlichen als kostengünstiges Signal dienen, um die Qualität einer Kandidatin oder eines Kandidaten in den verschiedenen betrieblichen Welten abzuschätzen. Im nachfolgenden Kapital wird daher der Frage nachgegangen, welche Konventionen sich im Kontext einer kulturell gesetzten Zweigeschlechtlichkeit dazu eignen könnten, dem Geschlecht als Auswahlkriterium betrieblichen Sinn zuzuweisen. Denn Bewerber und Ausbildungsplätze zu matchen erfordert immer auch, dass das Resultat der Selektion im einzelbetrieblichen Umfeld sinnvoll interpretierbar ist und vor den Mitarbeitenden, der Kundschaft und den Geschäftspartnern auf Akzeptanz stoßen 
wird. Betriebe sind daher auf geeignete Formen der Legitimation angewiesen, um ihre Personalentscheide bei Bedarf zu rechtfertigen.

Als zentrale Rechtfertigungsordnungen in Organisationen des Wirtschaftssystems wurden in der Forschungsliteratur bisher folgende Konventionen diskutiert (ausführlicher dazu Diaz-Bone 2009): die industrielle Konvention, die Marktkonvention und die häusliche Konvention. ${ }^{3}$ Aus Sicht des Ausbildungssystems gilt es zusätzlich die staatsbürgerliche Konvention (vgl. Imdorf und Leemann 2012) sowie das Koordinationsprinzip der Welt der Inspiration (Berufung) zu berücksichtigen.

\section{Die Relevanz des Geschlechts als Selektionskriterium}

Mit Bezugnahme auf die bisherige internationale Forschung zur Ausbildungsplatzvergabe wird im Folgenden die potenzielle Bedeutung des Geschlechts bei der Abschätzung von Koordinationserfordernissen in den verschiedenen Welten eines Ausbildungsbetriebs skizziert. Die Beschreibung der Welten orientiert sich an Diaz-Bone (2009) sowie an Imdorf und Leemann (2012).

\subsection{Frauenkörper als Anstellungshindernis in der industriellen Welt}

Die industrielle Konvention stärkt das Prinzip der Effizienz in der betrieblichen Handlungskoordination. Um Ressourcen möglichst produktiv zu nutzen, orientieren sich die Handlungsabläufe an einer effizienten Arbeitsorganisation mit planbaren Arbeitsbeziehungen. In dieser in ökonomischen Theorien wohlbekannten industriellen Welt, in der sich die Qualität der Mitarbeitenden an deren ,Produktivität' misst, ist bei der Selektion von Auszubildenden die Erwartung bedeutsam, dass sie die künftigen Produktionsabläufe aufgrund ihrer Fähigkeiten, Fertigkeiten und körperlichen Voraussetzung unterstützen und nicht stören werden. Anderweitige industrielle Koordinationsanforderungen, an denen Ausbildungsplatzanwärter und ihre ,Arbeitsmoral' gemessen werden, sind Arbeitstugenden wie Ordnung, Sauberkeit, Pünktlichkeit, Regelmäßigkeit und Leistungsbereitschaft (vgl. auch Mariak und Kluge 1998: 297f.).

\footnotetext{
${ }^{3}$ Auf die Darstellung der Netzwerkkonvention der projektförmigen Welt, einer vierten, in modernen Arbeitsorganisationen relevanten Konvention, wird hier verzichtet, da sich diese Konvention im untersuchten Autogewerbe für das Verständnis der Geschlechterselektion nicht als relevant erwiesen hat.
} 
Hinsichtlich der Bedeutung, die dem Geschlecht in der industriellen Welt zukommt, stellt sich die Frage, inwiefern dieses für Produktivitätsannahmen als relevant erachtet wird. In den bisherigen Forschungsbefunden wird vor allem auf die Bedeutung der Körper von Frauen und Männern für deren Funktionstüchtigkeit in der industriellen Welt verwiesen. Sowohl in den deutschen Untersuchungen von Rauch und Schober (1996: 26) sowie Mariak und Kluge (1998: 210) als auch in englischen Studien (Ashton und Maguire 1980: 117; Fuller et al. 2005: 304; Miller et al. 2005: 61) werden ,Männerberufe' von den Selektionsverantwortlichen mit physischer Voraussetzung und Belastbarkeit assoziiert, welche Männer eher erfüllen würden als Frauen. Argumente wie unzureichendes technisches Verständnis oder mangelnde handwerkliche bzw. manuelle Fertigkeiten spielen dagegen in den Begründungen für die Ablehnung von weiblichen Arbeitskräften für männertypische Tätigkeiten bei Rauch und Schober (1996: 28) nur eine untergeordnete Rolle.

\section{2 Ökonomisierung von Geschlecht in der Welt des Marktes}

In Abgrenzung zur effizienzbasierten Koordinationsordnung der industriellen Welt hebt die Marktkonvention die Beziehungsprinzipien des Preises und der Konkurrenz hervor. In der Welt des Marktes sind soziale Beziehungen strategisch und opportunistisch. Eine Mitarbeiterin erhält Qualität, wenn sie für den Betrieb ein nachgefragtes Gut mit knappem Angebot bedeutet. Für die Ausbildungsstellenvergabe bedeutet die Marktkonvention, dass dem Betrieb durch einen künftigen Auszubildenden keine unnötigen Kosten entstehen. Betriebe wollen insbesondere keine Jugendlichen einstellen, die ihre Ausbildung frühzeitig abbrechen könnten, da dies einer betrieblichen Fehlinvestition gleichkäme.

Im Hinblick auf die Erfordernisse dieser Welt des Marktes gibt die Forschungsliteratur zu männertypischen Berufen Hinweise auf eine die Frauen diskriminierende Ökonomisierung von Geschlecht bei der Ausbildungsplatzvergabe. Mariak und Kluge (1998: 209f) verweisen auf die Erwartung von Arbeitgebern, dass weibliche Auszubildende nach ihrer erfolgreichen Ausbildung aufgrund der traditionellen Rollenteilung häufiger den Betrieb wechseln oder den Beruf aufgeben könnten. Damit seien die Kosten ihrer Ausbildung nicht mehr durch Gewinne aus ihrer Facharbeit aufzufangen und Frauen würden zu einem Investitionsrisiko. Dass die Arbeitgeber bei Schulabgängerinnen eine ökonomisch unvorteilhafte, schwangerschaftsbedingte Unterbrechung der Ausbildungsjahre antizipieren könnten, 
ist in der Schweiz vor dem Hintergrund der tiefen Geburtenrate im Jugendalter $(0,4$ Geburten auf 1000 weibliche Jugendliche, vgl. Michaud 2003) allerdings wenig plausibel.

In mehreren Untersuchungen findet sich hingegen eine plausiblere, gegen weibliche Kandidatinnen gerichtete Argumentation, wonach die verfügbaren sanitären Anlagen in manchem Betrieb mit männlicher Belegschaft keine separaten Wasch- und Umkleidemöglichkeiten für Frauen böten (Fuller et al. 2005: 304; Mariak und Kluge 1998: 210; Rauch und Schober 1996: 26). Diese Rechtfertigung ist auch in Schweizer Ausbildungsbetrieben denkbar, da das Arbeitsgesetz in Betrieben mit mehr als fünf Mitarbeitenden und bei „,verschmutzender Tätigkeit“ nach Geschlechtern getrennte Garderoben, Waschanlagen und Toiletten verlangt (SECO o.J.: 329-2). Sobald die Schaffung solcher Sozialräume mit einem betrieblich nicht vertretbaren Aufwand verbunden wird (so bei Mariak und Kluge 1998: 210), lässt sich der Ausschluss von Frauen mit Bezugnahme auf die Konvention des Marktes legitimieren.

\subsection{Traditionelle Geschlechterverhältnisse in der häuslichen Welt}

Basierend auf der häuslichen Konvention erlangt eine Person ,Qualität', wenn ihr aufgrund von sozialer Nähe, Bekanntschaft, Verwandtschaft oder persönlicher Beziehungen Vertrauen entgegengebracht wird. Die Beziehungserwartungen orientieren sich an den Koordinationsformen der traditionell strukturierten Familie, etwa an altersspezifischen Autoritäts- und Abhängigkeitsverhältnissen sowie - für die vorliegende Thematik besonders relevant - an wertkonservativen Geschlechterrollen, an einer traditionellen geschlechtlichen Arbeitsteilung und an einer heterosexuellen Norm von Paar- und Geschlechterbeziehungen. Betriebe sind bei der Selektion von Auszubildenden in der häuslichen Welt bestrebt, konfliktarme horizontale und vertikale Sozialbeziehungen zu generieren. Eine auf häuslichen Werten basierende soziale Kohäsion im Betrieb könnte neben Altersdifferenzen (Imdorf 2012) und ethnischer Heterogenität (Imdorf 2010a) durch ungewohnte Geschlechterverhältnisse in Frage gestellt werden. Die Ausbildungsverantwortlichen in männertypischen Branchen könnten bei Bewerbungen junger Frauen somit eine Störung der innerbetrieblichen Sozialbeziehungen antizipieren.

Die Forschungsliteratur gibt dazu nur spärliche Hinweise. Fuller et al. (2005: 305) zeigen in ihrer englischen Untersuchung, dass die Arbeitgeber in Branchen wie Bau, Klempnerei oder Maschinenbau aus Tradition das ,gewohnte Geschlecht' ausbilden. 
Die Autorinnen zitieren einen Klempner, der angab, dass die männliche Arbeitsumgebung und die Einstellungen der Arbeiter gegenüber Frauen verändert werden müssten, damit der Beruf auch für Frauen zugänglich werden würde. Er hob die rüden Umgangsformen am Arbeitsplatz (Gefluche und sexuelle Anzüglichkeiten) hervor, die auch von ihm erwartet wurden, und die er sich selbst in seinen ersten Arbeitsjahren angeeignet habe (ebd.). Ashton und Maguire (1980: 117) verweisen zudem auf den arbeitgeberseitigen Glauben, wonach junge Frauen dem Verhalten, den Einstellungen und der Sprache einer männlichen Arbeitsgruppe nicht gewachsen seien.

\subsection{Geschlecht in der staatsbürgerlichen Welt und in der Welt der Inspiration}

Zusätzlich zu den bisher ausgeführten drei Konventionen der Arbeit gilt es die für das Ausbildungssystem zentrale staatsbürgerliche Konvention zu berücksichtigen. Sie reklamiert einen Gesellschaftsvertrag, der bei der Gestaltung von Beziehungen das Prinzip der Chancengleichheit in den Vordergrund rückt. Im Hinblick auf die Geschlechterkategorie wären die Betriebe in der staatsbürgerlichen Welt aufgefordert, ihre Ausbildungsplätze chancengleich an männliche und weibliche Bewerber zu vergeben. Während Großbetriebe teilweise entsprechende Vorkehrungen treffen (Gender-Mainstreaming in der Personalpolitik), strukturiert die staatsbürgerliche Konvention die Vergabe von Ausbildungsplätzen im Kleingewerbe nur schwach, da der Staat die Lehrlingsauswahl (im Unterschied zur Gestaltung des Ausbildungsverhältnisses) kaum reguliert.

Schließlich lässt sich die von Boltanski und Thévenot (2007: 225) beschriebene Welt der Inspiration bezüglich der Bedeutung des Geschlechts bei der Ausbildungsplatzvergabe hinterfragen. In dieser Welt ist der soziale Zusammenhalt einer Berufsgruppe durch das gemeinsame Sich-berufen-fühlen gewährleistet. Bei der beruflichen Motivation und den beruflichen Interessen handelt es sich um eine in Ausbildungsbetrieben besonders hoch bewertete Qualität, der im Selektionsprozess besondere Aufmerksamkeit geschenkt wird. Mariak und Kluge (1998) haben auf die besondere ,Berufung' von Mädchen mit atypischen Berufsorientierungen für männertypische Berufe hingewiesen. Die in ihrer Studie befragten Berufsausbilder waren einhellig der Meinung, dass weibliche Auszubildende in männertypischen

Branchen mindestens genauso gut seien wie männliche. Fachfrauen in ,Männerberufen' würden zudem einen besonderen Ehrgeiz besitzen und möchten 
männlichen Kollegen nicht nachstehen (ebd.: 209). Damit könnten sie das Interesse von Ausbildungsbetrieben in männertypischen Branchen wecken.

\subsection{Forschungsfrage}

Die skizzierten Konventionen können als wesentliche Beziehungs- und Koordinationsprinzipien von Ausbildungsorganisationen betrachtet werden. Sie gewährleisten die Abstimmung der gegenseitigen Erwartungen im Betrieb und leiten die Beurteilung der Bewerber bei der Ausbildungsplatzvergabe an. Wie alle anderen Auswahlkriterien lässt sich auch das Individualmerkmal Geschlecht vor dem Hintergrund solcher Konventionen interpretieren. Die verschiedenen Welten zeichnen sich dabei durch eine unterschiedliche Bedeutung und Anerkennungslogik von Geschlecht für den betrieblichen Zusammenhalt aus.

Im Folgenden soll mit einer qualitativen Studie aus der Schweiz geklärt werden, welche ausbildungsrelevanten Risiken und Qualitäten mit dem weiblichen Geschlecht in einer männertypischen Berufsbranche, dem Autogewerbe, in Verbindung gebracht werden. Wie rechtfertigen die Arbeitgeber ihre geschlechtertypischen Präferenzen? Welche zukünftigen konfliktträchtigen (Ausbildungs-)Situationen werden aufgrund der Geschlechterzugehörigkeit der Kandidatinnen und Kandidaten antizipiert? Wie lassen sich solche Situationen und die darin eingelassenen Motive den skizzierten Welten und Konventionen zuordnen? Falls sich eine Geschlechterrelevanz der für die Personalselektion maßgeblichen Konventionen aufzeigen lässt, dann verweist dies auf betriebliche Motive, die den Ausschluss von weiblichen Auszubildenden im Autogewerbe bewirken und so die Geschlechtersegregation in dieser Branche mitbedingen.

\section{Daten und Methode}

Zur Beantwortung der Forschungsfrage wird auf die Daten des Schweizer Forschungsprojekts ,Lehrlingsselektion in kleinen und mittleren Betrieben' aus dem Jahr 2005 zurückgegriffen ${ }^{4}$. Nachfolgend werden Stichprobe, Datensammlung und Auswertungsstrategie kurz umrissen. Ausführlichere Angaben zum methodischen Vorgehen finden sich im Methodenbericht der Studie (Imdorf 2010b).

\footnotetext{
${ }^{4}$ Das im Nationalen Forschungsprogramm ,Integration und Ausschluss' durch den Schweizerischen Nationalfonds geförderte Projekt am Heilpädagogischen Institut der Universität Fribourg untersuchte in der Zeit von 2004 - 2006 die betrieblichen Logiken bei der Besetzung beruflicher Ausbildungsplätze in Deutschschweizer Betrieben.
} 


\subsection{Untersuchte Betriebe}

Die Analyse bezieht sich auf Deutschschweizer Ausbildungsbetriebe des Auto- und Karosseriegewerbes. Die Betriebe kleiner und mittlerer Größe wurden indirekt über das Nachverfolgen einzelner Bewerbungen von Jugendlichen rekrutiert, die sich ohne Erfolg auf ausgewählte Ausbildungsberufe (hier: Automechaniker/in, Automonteur/in oder Autolackierer/in) beworben hatten. Es konnten Daten aus 27 Betrieben ausgewertet werden: 17 Autogaragen und 10 Karosserie- oder Autospritzwerke. 21 der 27 Betriebe sind Kleinbetriebe mit max. 50 Angestellten. Unter den 27 Interviewpartnern war nur eine Frau (die Personalfachfrau eines größeren Garagenbetriebs), was die berufsbildungsstatistisch bekannte marginale Vertretung von Frauen im Auto- und Karosseriegewerbe widerspiegelt. Eine Übersicht über die untersuchten Betriebe nach Art, Anzahl Mitarbeitenden, betrieblicher Position des Interviewpartners und Ausbildungsberuf findet sich in Tabelle $1 \mathrm{im}$ Anhang.

\subsection{Datenerhebung}

In den rekrutierten Ausbildungsbetrieben führte der Autor mit den Selektionsverantwortlichen halbstrukturierte Experteninterviews durch. Der Interviewleitfaden umfasste detaillierte Fragen zur betriebsüblichen Lehrstellenvergabe. In diesem Zusammenhang wurde gefragt, welche Voraussetzungen eine erfolgreiche Bewerbung erfüllen muss und weshalb diese Voraussetzungen für den Betrieb von Bedeutung sind. Das Geschlecht als Auswahlkriterium wurde nicht direkt erfragt, aber zwei Mal als mögliche Deutungsressource angeboten; das erste Mal bei der Erfragung des Geschlechterverhältnisses des Bewerberpools, das zweite Mal mit einer Nachfrage gegen Ende des Interviews, ob die von den Interviewpartnern genannten Selektionskriterien geschlechtsspezifisch seien.

\subsection{Datenanalyse}

Aus der retrospektiven Befragung zu betrieblichen Selektionsprozessen resultieren Rechtfertigungen der Selektionsverantwortlichen. Die theoretische Prämisse, wonach Organisationen in der Regel Entscheide treffen, die sie später in ihrer Umwelt rechtfertigen können, impliziert eine Abhängigkeit des nachträglichen Legitimationshandelns von der realen Entscheidungssituation. Damit lässt sich von 
den empirisch greif- und analysierbaren Rechtfertigungsreden auf vergangene Entscheidungsprozesse schließen. Die transkribierten Interviews wurden zunächst deduktiv mittels geschlechterrelevanten Suchbegriffen nach Textstellen codiert, die im Hinblick auf die Fragestellungen bedeutsam waren. Die darin enthaltenen Rechtfertigungsreden wurden sodann einer Argumentationsanalyse unterzogen.

Die Argumentationsanalyse ist hier die Methode der Wahl, da Antworten auf die Frage, weshalb das Geschlecht bei der Ausbildungsplatzvergabe als wichtig erachtet wird, im Modus von Argumentationen gegeben werden. Damit lässt sich methodisch klären, wie von antizipierten Ausbildungssituationen, in denen Geschlecht als relevant für eine gelingende Koordination erachtet wird, auf die Eignung einer geschlechtlich klassifizierten Person für die Ausbildung im Betrieb geschlossen wird. Jeder Schlussfolgerung liegt dabei eine selektionsleitende Konvention (oder ein Kompromiss mehrerer Konventionen) zugrunde, die argumentationsanalytisch rekonstruiert werden können (ausführlich dazu vgl. Imdorf 2010b).

\section{Resultate}

\subsection{Bewerberpool und Geschlechterpräferenzen in den untersuchten Betrieben}

Für 16 der 27 untersuchten Betriebe konnten Angaben zum Geschlechterverhältnis des Bewerberpools ausgewertet werden. Zwei Garagenbetriebe gaben an, für den Ausbildungsberuf Automonteur/in bzw. -mechaniker/in keine Bewerbungen von Frauen erhalten zu haben. In vier Garagenbetrieben und in einer Lackiererei haben sich Frauen vereinzelt beworben. In drei Lackierereien war von „einigen“ Frauen die Rede; zwei gaben den Frauenanteil mit „zehn bis zwanzig Prozent“ an und zwei wiesen auf eine Zunahme von Bewerbungen durch Frauen hin. Zwei Betriebe, darunter ein Garagenbetrieb, berichteten von auffällig vielen Bewerbungen von Frauen. Die Angaben belegen, dass die Mehrheit der untersuchten Betriebe Bewerbungen von Frauen erhalten hatte.

20 der 27 untersuchten Betriebe hatten bereits Erfahrung mit der Ausbildung von Frauen, darunter auch überraschend viele Autogaragen. In zwei weitere Garagen und in einer Lackiererei wurden Mädchen schon zu Betriebspraktika zugelassen. Drei Autogaragen und eine Lackiererei gaben an, bisher keine Erfahrung mit weiblichen Auszubildenden zu haben.

In 26 von 27 Betrieben waren männliche Auszubildende willkommen. Dagegen gaben nur die Hälfte der Betriebe Präferenzen für weibliche Auszubildende an, wobei 
diesbezüglich kein Unterschied zwischen den Autogaragen und den Lackierereien zu konstatieren ist. Ein Garagenbetrieb [1] ${ }^{5}$ setzte ausschließlich auf die Ausbildung einer Monteurin oder Mechanikerin. Sechs Betriebe (darunter vier Garagenbetriebe) sprachen sich explizit gegen weibliche Auszubildende aus. Je zwei Garagenbetriebe bzw. Lackierereien äußerten sich ambivalent zu ihren Präferenzen, und in drei Garagenbetrieben blieb das Urteil unklar.

\subsection{Argumente gegen weibliche Auszubildende im Autogewerbe}

Im Folgenden werden die wesentlichen Argumente resümiert, mit denen die Arbeitgeber ihre Präferenzen gegen Frauen legitimiert haben. Dabei interessiert, welche Art konfliktträchtiger Situationen die Betriebe antizipieren, wenn sie weibliche Auszubildende einstellen würden, und in welchen der oben beschriebenen Welten die Konflikte verortet werden können.

Mit dem weiblichen Geschlecht assoziieren manche Ausbildungsverantwortliche körperliche Merkmale, die einen Arbeitsprozess behindern könnten. In zwei Betrieben $[2,3]$ wurde die menstruationsbedingt wiederkehrende Abwesenheit und Launenhaftigkeit früherer Mitarbeiterinnen bemängelt. Der Geschäftsinhaber [3] sah das wesentliche körperliche Problem jedoch primär in dem im Vergleich zu Männern schwächeren Körperbau der Frauen, was zu Rückenschmerzen führen könne, „wenn sie dann eine Woche lang Räder wechseln (...) und alles auf Brusthöhe heben müssen". Der Werkstattleiter [4] meinte, dass einem Mann die Körperkraft zugute käme, "wenn er ein Getriebe reinmacht", und in der Autolackiererei [5] hieß es, dass Autolackierer „nicht UNBEDINGT ${ }^{6}$ ein Beruf ist für eine Frau, wenn sie nicht ziemlich robust ist". Solche betrieblichen Stellungnahmen verweisen auf den Konfliktgehalt des Frauenkörpers in der industriellen Welt von Autogaragen: Weibliche Körper stören, so die Annahme einiger Garagisten, potenziell Arbeitsabläufe in der Werkstatt, die ein gewisses Maß an Körperkraft erfordern.

Andere Argumente gegen weibliche Auszubildende beziehen sich auf ein konfliktuelles Beziehungsgefüge innerhalb der Belegschaft, und damit auf eine Bedrohung des sozialen Zusammenhalts im Betrieb. Ein Filialleiter [6] verwies auf

\footnotetext{
${ }^{5}$ Ziffern in eckigen Zahlen [Betriebsnummer] dienen nachfolgend der Zuordnung zu den in Tabelle 1 im Anhang aufgelisteten Betrieben.

${ }^{6}$ Wörter, die in den Interviews besonders betont wurden, sind in den vorliegenden Interviewzitaten mit Großbuchstaben wiedergegeben.
} 
sein Team, das "besorgt (war), weil es eigentlich so ein wenig unter sich bleiben wollte”. Ein Lehrlingsausbildner [7] sah vor allem „ein Problem (...), wenn sie zu hübsch sind“, weil junge Frauen dann die Mechaniker ,ablenken“ und so den Betrieb stören könnten. Auch ein Geschäftsinhaber [8] verwies auf die Unruhe, die eine junge Frau in den Betrieb hineintragen könnte:

„,Dann gibt es immer hier so Macho-Zeugs mit den Jungs untereinander. Also wenn man junge Männer hat, das gibt immer solche Machtkämpfchen, und irgendeiner gewinnt dann. (...) Das kommt nicht gut, also das ZWISCHENMENSCHLICHE ist nicht gut. (...) das bringt sehr viel Unruhe (...).“

Eine Personalfachfrau [9] bestätigte vergleichbare Probleme, wonach einige Männer bereits den „Lehrtöchtern nachgestiegen“ wären. Anderswo wurde konstatiert, dass es nicht einfacher sei, „ein Mädchen auszubilden in einer Männerdomäne als nur Männer" [10].

In drei Betrieben [2, 10, 11] wurde zudem das raue Arbeitsklima betont, das im männertypischen Autogewerbe verbreitet sei. Es herrsche „ein rauer Ton“, es würde „nicht gehätschelt“ [2], die Umgangssprache sei „nicht so wie vielleicht auf einem Büro“ [11], eine Auszubildende dürfe „kein Mimöschen sein“, und man wisse daher nicht, „ob hier die Mädchen am richtigen Ort“ seien [2]. In zwei weiteren Betrieben $[4,12]$ wurde darauf hingewiesen, dass junge Frauen durch laute Zurechtweisungen überfordert würden. Der Werkstattleiter [4] wies das Problem dabei lachend von sich und hob die Gefährdung der psychischen Integrität von Frauen im Autogewerbe hervor:

"Also wir sind offen, also das ist kein Problem. Wir sind Jungs hier, es ist, es ist ein bisschen ein Problem für die Frau, (...) wenn es irgendwie Stressprobleme gibt, (...) die Frauen, die kommen in Stresssituationen, und es ist ihnen nicht wohl dabei. (...) ich habe einfach die Erfahrung (gemacht), und dann hat sie psychische Probleme bekommen.”

Betriebliche Problemerwartungen wie die Antizipation von Unruhe, wenn eine Frau zu einem männlichen Team stößt, das raue Arbeitsklima und die Gefährdung der psychischen Integrität von Frauen sind Argumente, die auf mögliche soziale und persönliche Konflikte in der häuslichen Welt der Betriebe verweisen.

Des Weiteren wurde in mehreren Betrieben mit dem Argument gegen Frauen votiert, dass diese die Ausbildung abbrechen oder den Beruf frühzeitig verlassen könnten. 
Der Geschäftsführer [2] gab an, dass man keine Mädchen mehr einstellen würde, nachdem eine Auszubildende ,nach kürzester Zeit schwanger (wurde) und mit dem Beruf aufgehört" habe. In der Autolackiererei [13], wo man weiblichen Auszubildenden durchaus nicht abgeneigt sei, werden Bewerbungen von Frauen besonders genau unter die Lupe genommen, denn man wolle „einen Lehrabbruch (...) auf jeden Fall verhindern". In drei weiteren Fällen [7, 8, 11] wurde problematisiert, dass Frauen mittelfristig nicht im gelernten Beruf verbleiben würden. In der Autogarage [7] wurde der interviewte Lehrlingsausbildner von seinem Chef angehalten, dem männlichen Konkurrenten gegenüber einer valablen weiblichen Kandidatin den Vorrang zu geben. Der Vorgesetzte hätte gemeint, dass beim männlichen Kandidaten nach der Lehre die Chance größere wäre, dass er dem Betrieb erhalten bliebe, da der Mann die Stelle brauche, um seine Familie zu ernähren. In diesem Fall erwies sich eine traditionelle Werthaltung hinsichtlich der familiären Arbeitsteilung, die sich am Modell des männlichen Familienernährers ausrichtet, als Grundlage der betrieblichen Kosten-Nutzen-Rechnung. Wenn betriebliche Investitionen in weibliche Auszubildende angesichts eines frühzeitigen Ausbildungsabbruchs oder eines ungesicherten Verbleibs in Beruf und Betrieb nicht lohnenswert erscheinen, kann das weibliche Geschlecht in der marktförmigen Welt der Ausbildungsbetriebe zu einem Ausschlussgrund werden.

Schließlich argumentierten die Selektionsverantwortlichen in vier Betrieben $[6,8,14$, 15] mit dem aus der Forschungsliteratur bekannten Argument, wonach fehlende Sozialräume bzw. Toiletten die Integration einer Frau in eine männliche Belegschaft nicht gestatten würden. Dabei wurden weniger die Kosten getrennter Sozialräume als vielmehr beschränkte räumliche Möglichkeiten hervorgehoben. In der Autolackiererei [8] wird jungen Frauen sofort abgesagt, denn man müsse ,eigentlich immer zwei WCs haben" und man habe "schlichtweg keinen Platz hier". Solange primär räumliche oder bauliche Beschränkungen betont werden, lassen sich solche Rechtfertigungen der industriellen Welt zuordnen. Sobald hingegen die gesetzliche Erfordernis nach geschlechtergetrennten Wasch- und Umziehräumen in den Vordergrund gerückt wird, argumentieren die Ausbildner in der staatsbürgerlichen Welt, in der das Arbeitsrecht die Integrität weiblicher Mitarbeiterinnen vor männlichen Zumutungen schützt. Diese Argumentation vertrat der Inhaber einer Autogarage [14], der betonte, dass man gerade am umbauen sei und in Zukunft ein separates WC haben werde. Dass die staatsbürgerliche Konvention durchaus den 
Referenzrahmen der Rechtfertigung darstellen kann, belegen drei Betriebe $[1,10,16]$, die betonten, dass man für weibliche Auszubildende eingerichtet sei und über die erforderlichen sanitären Einrichtungen verfüge.

Die rekonstruierten Rechtfertigungsreden gegen junge Frauen im männertypischen Beruf replizieren neben dem Sozialraumargument weitere aus der Forschungsliteratur bekannten Begründungen in der industriellen Welt (Körperkraft) und in der marktförmigen Welt (Verbleib im Beruf). Die betrieblichen Stellungnahmen verweisen darüber hinaus deutlich auf zusätzliche beträchtliche Hürden junger Frauen im Zugang zur häuslichen Welt der männertypischen Autoreparaturbranche.

\subsection{Argumente zu Gunsten weiblicher Auszubildender im Autogewerbe}

Trotz der statistisch randständigen Vertretung junger Frauen insbesondere im Autoreparaturgewerbe befürwortete immerhin die Hälfte der untersuchten Betriebe die Einstellung von weiblichen Auszubildenden. Diese Betriebe hoben insbesondere die Motivation von weiblichen Auszubildenden, ihre guten Leistungen in Berufsschule und Betrieb sowie deren positiven Einfluss auf das Betriebsklima hervor, Qualitäten also, die sich konfliktreduzierend auf ein Ausbildungsverhältnis auswirken.

Bemerkenswert ist die Beobachtung in den Betrieben, wonach Interesse und Motivation weiblicher Auszubildender für die untersuchten Berufe im Vergleich zu ihren männlichen Kollegen besonders ausgeprägt seien. "Diese Mädels, die das machen, das sind solche, die dann hinter dem Beruf stehen" [17]. Ein Geschäftsinhaber [1] lobte das Motivationsschreiben einer weiblichen Kandidatin: „Sie hat in handwerklichen Berufen geschnuppert, NUR handwerkliche Berufe“. Er zitierte die Kandidatin aus den Unterlagen: „,Ich werke auch gerne mit meinen Händen und mit Metall (...)'. Die Anderen [die Jungen] schreiben: 'Ich habe Freude an Autos'. Ha ha, da bekomme ich ja Zustände”. Ein Mädchen wisse, was es wolle, wenn es ,in einen absoluten Männerberuf einbricht“, meinte der Geschäftsinhaber [18], der sich überzeugt zeigte, ,dass die MEHR wissen, was sie machen als mancher Junge". Mit solchen Aussagen rechtfertigen die Betriebe die motivationale Qualität junger Frauen und damit ihre ,Berufung' in der Welt der Inspiration.

Zudem wurden die guten Leistungen weiblicher Auszubildender in Berufsschule und Betrieb betont. Ihre Schulleistungen fallen zum einen in den Bewerbungsunterlagen positiv auf. Laut einem interviewten Geschäftsinhaber [14] waren „ALLE 
Bewerbungen von denen [den Mädchen] mit Abstand die Besten”. Auch die in der Autolackiererei [13] neu eingestellte Auszubildende war „,von der Qualifikation her“ die Beste. Die guten Schulleistungen bestätigen sich zum anderen in der Berufsschule. Ein Lehrlingsverantwortlicher [15], der selbst unterrichtet, berichtete von zwei Frauen in seiner Klasse, die „mit den Noten (...) im oberen Viertel waren“. Auch in der Garage [19] war die Rede von Mädchen mit „guten bis sehr guten Noten“. In zwei Interviews wurde darüber hinaus der positive Einfluss der Mädchen auf die Leistungsmotivation in der Schulklasse hervorgehoben. „Sie war natürlich dort [in der Berufsschule] schon ein wenig der Reißer (...), äh sie war dann eben auch der Gradmesser eben in der Schule. Und die Jungs hat das dann natürlich angespornt“ [20]. "Wenn es Mädchen in der Klasse hat, dann hört man, dann ist der Klassenschnitt höher" [21]. Gute schulische Leistungsprognosen sind für Ausbildungsbetriebe primär relevant, weil damit das Risiko eines berufsschulisch bedingten Ausbildungsabbruchs sinkt (Imdorf 2009). Deshalb lassen sich die auf berufsschulischen Erfolg gerichteten Rechtfertigungsreden in der Regel in der marktförmigen Welt verorten.

Neben der schulischen Leistungsstärke wird jungen Frauen aber auch ein gutes Leistungsverhalten im Betrieb zugeschrieben. Das Argument der körperlichen Unzulänglichkeiten wird dabei relativiert. “So streng ist der Job ja nicht” [21]; „Es gibt sicher gewisse Sachen (...) die vielleicht rein von der Kraft her nicht mehr gehen (...), das kann man umgehen, (die sind) für mich (...) vernachlässigbar” [16]. Ein Geschäftsinhaber [1] verwies auf eine Auszubildende, die mangelnde Körperkraft mit einer intelligenten Arbeitsorganisation kompensieren konnte: „Man kann natürlich, wenn man (...) ein wenig clever arbeitet, kann man das sehr angenehm einrichten. (Aber) man kann die Kilos (auch) um das Dorf tragen, wenn man will." Hervorgehoben wurden vielmehr die Qualitäten weiblicher Auszubildender in der industriellen Welt: Gelobt wurde etwa ihre Zuverlässigkeit und ihr Verantwortungsbewusstsein [7], das „technische Flair“ von „Mädels, die das machen“ [17], oder - in zwei Autolackierereien [21, 22] - das besondere „Gefühl für die Farben“ von Frauen. Sie würden zudem „sauber“ [4, 7] und „,seriöser“ [23] arbeiten. Solcherart fachliche Qualifikationen würden auch anlässlich von Lehrabschlussprüfungen und nationalen Berufswettbewerben unter Beweis gestellt: „Sie hat sensationell abgeschnitten bei der Schweizermeisterschaft“ [18]; „Sie machte die beste Prüfung im Kanton, (...) sie war in der Vorausscheidung der Berufsolympiade“ [20]. 
Schließlich hoben einige Ausbildungsverantwortliche die Qualitäten weiblicher Auszubildender im sozialen Arbeitszusammenhang hervor. Frauen wurden als teamfähig und „sehr anpassend“ [22] wahrgenommen. Anfängliche Zweifel hinsichtlich der Zusammenarbeit im Betrieb konnten in der Autogarage [17] aufgrund der Erfahrung mit einer weiblichen Hilfskraft gemindert werden. Auch in der Autolackiererei [24] konnte das Team in Zusammenarbeit mit einer ausgebildeten Autolackiererin die Erfahrung machen, ,dass eine Frau nicht stört““. Im Gegenteil, in mehreren Betrieben wurde auf den positiven Einfluss weiblicher Mitarbeitender auf das Betriebsklima hingewiesen. Es beruhige "unheimlich das Klima in einem Betrieb, wenn man eine Frau oder ein Mädchen hier hat” [12]. Es sei „einfach ein wenig ein anderes Gefühl in der Firma”, und auch die Umgangssprache sei eine andere. Der Abteilungsleiter [10] bestätigte, dass seine männlichen Mitarbeiter bei Anwesenheit einer Frau „weniger harte Wörter brauchen“ würden. Für sich selbst sah er zudem den Vorteil, dass er bei Anwesenheit einer Frau einen Grund habe, um vorlaute Mitarbeiter zurechtzuweisen. In der Autogarage [1] hatte eine Praktikantin „das ganze Klima verfeinert“" (,sie hat Leben gebracht, sie hat ein Strahlen gebracht"). In einer weiteren Autogarage [19] war die Rede davon, dass eine Frau dem Betrieb einen „Familiensinn“ geben würde, indem die männlichen Kollegen dazu angehalten würden, ihr zu helfen. „Unter dem Strich“, so das Urteil, ,würde es dem Betrieb etwas bringen“. Offensichtlich führt die Präsenz einer jungen Frau in der häuslichen Welt von Männerbetrieben auch zu Harmonie und nicht notgedrungen zu sozialer Unruhe.

Die skizzierten Rechtfertigungen lassen auf Vorteile für das Autogewerbe durch die Inklusion weiblicher Mitarbeiterinnen schließen. Dabei fällt auf, dass die Betriebe die Integration weiblicher Auszubildender in männertypische Berufe fast nie mit der Forderung nach Chancengleichheit von Frauen und Männern in der staatsbürgerlichen Welt legitimiert haben.

\section{Zusammenfassung und praktische Implikationen}

Mittels einer konventionensoziologischen Analyse wurde die betriebliche Bedeutung des zugeschriebenen weiblichen Geschlechts bei der Auswahl von Auszubildenden im männertypischen Autogewerbe anschaulich verdeutlicht. Im Kontext von kulturell gesetzter Zweigeschlechtlichkeit hat die Analyse den betrieblichen Sinn der ,Organisationsressource' Geschlecht bei der Personalauswahl offen gelegt, der in der 
Antizipation von konfliktträchtigen oder harmonieförderlichen Situationen des künftigen Ausbildungsverhältnisses besteht. Betriebe, wählen' bei der Lehrlingsauswahl mitunter Geschlecht, um auf ein möglichst störungsfreies Ausbildungsverhältnis hinzuwirken.

Vorausgesetzt, die vorliegenden Befunde lassen sich in weiteren geschlechtertypischen Branchen replizieren, lässt sich die These stützen, dass die Geschlechtersegregation auf dem Ausbildungsmarkt auch durch betriebsseitige Selektionskalküle verfestigt wird (vgl. bereits Mariak und Kluge 1998: 211). Die konventionentheoretische Auslegeordnung der betrieblichen Argumente für und wider weibliche Auszubildende im Autogewerbe belegt dabei sowohl klassische, aus der Forschungsliteratur bereits bekannte Argumente ihres Ausschlusses als auch neue Argumente, die vermehrt für die betriebliche Integration junger Frauen im untersuchten Gewerbe sprechen.

In der industriellen Welt der Betriebe konkurrieren traditionelle Rechtfertigungen, die jungen Frauen die für die Berufsausübung erforderliche Körperkraft absprechen, mit Rechtfertigungsreden, die dieses Argument zurückweisen und die Leistungsfähigkeit und -bereitschaft von Frauen im Betrieb hervorheben. In der häuslichen Welt stehen Befürchtungen der mangelnden sozialen Passung und die Figur der sozialen Unruhestifterin wiederum Hoffnungen gegenüber, wonach Frauen dem sozialen Betriebsklima förderlich sein könnten. In der Welt des Marktes konkurrieren Bedenken, dass die ausgebildeten Frauen den Beruf frühzeitig verlassen könnten, mit der Gewissheit, dass bei weiblichen Auszubildenden kein Ausbildungsabbruch infolge schulischer Probleme zu erwarten ist. Dass die marktförmige Konvention die Integration junger Frauen ins männertypische Gewerbe zusätzlich befördern kann, belegen Forschungsbefunde, wonach sich die Arbeitgeber in technischen Branchen in Zeiten zunehmenden Fachkräftemangels und rückläufigen Interesses des männlichen Nachwuchses vermehrt für weibliche Auszubildende stark machen (Lemarchant 2007: 61; Miller et al. 2005: 61). Auch in der staatsbürgerlichen Welt lässt sich für und gegen junge Frauen argumentieren: Das Gebot der Chancengleichheit sollte ihre Einstellung eigentlich befördern; der im Arbeitsgesetz verankerte Schutz vor sexueller Belästigung schließt sie dagegen in jenen Betrieben aus, die nicht über die erforderlichen sanitären Anlagen verfügen.

Im Hinblick auf die Integrierbarkeit junger Frauen in das männertypische Autogewerbe ist es daher weniger eine spezifische Konvention, die die Integration der 
Frauen besonders zu fördern vermag ${ }^{7}$. Es stellt sich vielmehr die Frage nach den Erfordernissen und Interessen innerhalb der einzelnen Welten: Starke Arme oder fachliche Kompetenz in der industriellen Welt; ungestörte Männerbünde oder die Förderung von Friedfertigkeit und Harmonie in der häuslichen Welt; längerfristige betrieblich-berufliche Bindung oder kurzfristiger Ausbildungserfolg in der Welt des Marktes. In der industriellen Welt dürfte das Argument der Fachkompetenz und der Leistungsbereitschaft jenes der Körperkraft, die sich heute durch technische Hilfsmittel kompensieren lässt, inzwischen übertrumpfen. In der Welt des Marktes könnten der steigende Fachkräftemangel sowie die zunehmenden Probleme der Betriebe, überhaupt Auszubildende zu rekrutieren, die Zugangschancen für weibliche Auszubildende erhöhen. In der häuslichen Welt wurde die Qualität weiblicher Auszubildender zumindest erkannt.

Dass den jungen Frauen der Einstieg in männertypische Branchen weiterhin schwer fällt, hängt möglicherweise damit zusammen, dass die traditionellen Ausschlussmechanismen der häuslichen Welt weiterwirken. Die analysierten Interviews belegen wohl eine betriebliche Nachfrage nach weiblichen Auszubildenden im Autogewerbe. Die maskuline Betriebskultur steht jedoch nicht zur Diskussion. Von den jungen Frauen wird erwartet, dass sie diese Kultur akzeptieren und erdulden. Sie werden im Rahmen von Betriebspraktika und Bewerbungsgesprächen explizit mit dieser Erfordernis konfrontiert: „Ich zähle denen beim Vorstellungsgespräch ganz klar alle Nachteile auf, die dieser Beruf hat für eine Frau“ [12]; „Weil es eben ein typischer Männerberuf ist, hat man sie auch deutlich darauf aufmerksam gemacht, (...) in WELCHEM Umfeld sie sich drei Jahre lang behaupten können muss" [13]; "Das habe ich ihr gesagt am Anfang: wenn sie wolle, dann müsse sie einfach kämpfen, als Frau” [23].

Diese Zumutung kann Bewerberinnen noch während des Selektionsprozesses zum Selbstausschluss bewegen, worauf folgende Beobachtungen hindeuten: „Häufig, wenn sie dann sehen, was sie alles können müssen, nach dem Eignungstest, sagen die von sich aus ,nein“" [11]; ,Sie kam am Montag (zum Testpraktikum), ging um zwölf Uhr, und nach dem Mittag [hat man sie] nie mehr gesehen oder gehört“ [19]. Damit können sich die geschlechteratypischen Aspirationen weiblicher Jugendlicher bereits

\footnotetext{
7 Hierin unterscheidet sich der betriebliche Ausschluss junger Frauen vom betrieblichen Ausländerausschluss. Während die industrielle Welt Jugendlichen gegenüber, die als ausländisch gelten, prinzipiell wohlgesinnt ist, wird ihnen der Zutritt in die häusliche und marktförmige Welt der Betriebe verwehrt (vgl. Imdorf 2010a).
} 
durch die Erfahrung des Bewerbungsprozesses abkühlen und die Betroffenen frühzeitig zum Selbstausschluss bewegen. Solche Abkühlungsprozesse gilt es vertiefter $\mathrm{zu}$ untersuchen, um $\mathrm{zu}$ klären, wie die jungen Frauen in ihren Bewerbungsbemühungen vermehrt unterstützt werden müssten.

Das analysierte Interviewmaterial ergibt schließlich auch Hinweise darauf, welche Ressourcen junge Frauen aus Sicht von Ausbildungsverantwortlichen benötigen, damit ihnen nicht nur der Bewerbungsprozess, sondern im Anschluss daran auch die Ausbildung im männertypischen Autogewerbe gelingt: Bewusstsein für die „Männerwelt“ im Betrieb [19, 25]; Wille, sich in diese zu integrieren [16]; Durchsetzungsvermögen [20]; psychische Stabilität und Belastbarkeit [25]; überdurchschnittliche Leistungsfähigkeit [20]; und die Unterstützungsbereitschaft der Eltern [13]. Eine wichtige Ressource ist zudem ein für die Geschlechterfrage sensibilisierter Ausbildner, der die jungen Frauen vor den Zumutungen männlicher Mitarbeiter zu schützen weiß [1, 13].

Für die Handlungspraxis implizieren die vorliegenden Ergebnisse, dass eine Förderung geschlechteratypischer Berufsaspirationen nicht genügt, um den Zugang junger Frauen in männertypische Berufe zu erreichen. Weibliche Auszubildende bedürfen zusätzlicher Ressourcen und Unterstützung, um nicht nur die Ausbildung, sondern bereits den betrieblichen Selektionsprozess in einer männertypischen Branche erfolgreich zu bewältigen. Der gewählte konventionensoziologische Analyserahmen impliziert jedoch zuallererst Interventionen, die bei den Ausbildungsbetrieben ansetzen, um die Integration weiblicher Auszubildender in der untersuchten Branche zu fördern. Branchenverbände können die herausgearbeiteten Pro-Argumente nutzen, um ihre Mitglieder zu überzeugen, vermehrt junge Frauen auszubilden. Den Betrieben wiederum kann das Argumentarium dazu dienen, die Einstellung weiblicher Auszubildender in ihrem lokalen Umfeld zu rechtfertigen. Es lohnt sich schließlich, darüber nachzudenken, wie die Betriebe ihre häusliche Welt umgestalten sollten, um die erwünschte Integration weiblicher Auszubildender nicht nur einzufordern, sondern erfolgreich umzusetzen.

\section{Literaturverzeichnis}

Ashton, David, und Malcolm Maguire. 1980. Young women in the labour market.

Stability and change. In Schooling for women's work, Hrsg. Rosemary Deem, 112125. London: Routledge \& Kegan Paul. 
BBT, Bundesamt für Berufsbildung und Technologie. 2011. Berufsbildung in der Schweiz 2011. Bern: Bundesamt für Berufsbildung und Technologie.

BFS, Bundesamt für Statistik. 2006. BFS Aktuell. Statistik der beruflichen Grundbildung 2005. Neuchâtel: Bundesamt für Statistik.

BMBF, Bundesministerium für Bildung und Forschung. 2003. Berufsbildungsbericht 2003. Bonn, Berlin: Bundesministerium für Bildung und Forschung.

Boltanski, Luc, und Laurent Thévenot. 2007. Über die Rechtfertigung. Eine Soziologie der kritischen Urteilskraft. Hamburg: Hamburger Edition.

Borkowsky, Anna. 2000. Frauen und Männer in der Berufsbildung der Schweiz. Schweizerische Zeitschrift für Bildungswissenschaften 22(2): 279-294.

Brandt, Oliver, und Waltraud Cornelißen. 2004. Berufsfindung in einer geschlechtercodierten Welt. Praxistheoretische Ansätze können der Berufsfindungsforschung neue Impulse geben. Zeitschrift für Frauenforschung und Geschlechterstudien 22(4): 21-38.

Diaz-Bone, Rainer. 2009. Konvention, Organisation und Institution. Der institutionentheoretische Beitrag der ,Économie des conventions‘. Historical Social Research 34(2): 235-264.

Fuller, Alison, Vanessa Beck und Lorna Unwin. 2005. The gendered nature of apprenticeship. Employers' and young people's perspectives. Education + Training 47(4/5): 298-311.

Gianettoni, Lavinia, Pierre Simon-Vermot, und Jacques-Antoine Gauthier. 2010. Orientations professionnelles atypiques: transgression des normes de genre et effets identitaires. Revue Française de Pédagogie 173: 41-50.

Imdorf, Christian. 2009. Die betriebliche Verwertung von Schulzeugnissen bei der Ausbildungsstellenvergabe. Empirische Pädagogik 23(4): 392-409.

Imdorf, Christian. 2010a. Die Diskriminierung ‘ausländischer’ Jugendlicher bei der Lehrlingsauswahl. In Diskriminierung. Grundlagen und Forschungsergebnisse, Hrsg. Ulrike Hormel und Albert Scherr, 197-219. Wiesbaden: VS Verlag für Sozialwissenschaften.

Imdorf, Christian. 2010b. Forschungsprojekt "Lehrlingsselektion in KMU”. Methodenbericht. Basel: Institut für Soziologie der Universität Basel.

Imdorf, Christian. 2012. Zu jung oder zu alt für eine Lehre? Altersdiskriminierung bei der Ausbildungsplatzvergabe. Journal for Labour Market Research 45(1): 79-98. 
Imdorf, Christian, und Regula J. Leemann. 2012. New models of apprenticeship and equal employment opportunity. Do training networks enhance fair hiring practices? Journal of Vocational Education \& Training 64(1): 57-74.

Leemann, Regula J., und Andrea Keck. 2005. Der Übergang von der Ausbildung in den Beruf. Die Bedeutung von Qualifikation, Generation und Geschlecht. Neuchâtel: Bundesamt für Statistik.

Lemarchant, Clotilde. 2007. La mixité inachevée. Garçons et filles minoritaires dans les filières techniques. Travail, genre et sociétés 18-2007: 47-64.

Mariak, Volker, und Susann Kluge. 1998. Zur Konstruktion des ordentlichen Menschen. Normierungen in Ausbildung und Beruf. Frankfurt a.M.: Verlag der Gesellschaft zur Förderung arbeitsorientierter Forschung und Bildung.

Meyer, Thomas, Barbara E. Stalder, und Monika Matter. 2003. Bildungswunsch und Wirklichkeit. Thematischer Bericht der Erhebung PISA 2000. Neuchâtel: BFS / EDK.

Michaud, Pierre-André. 2003. Prevention and Health Promotion in School and Community Settings: A Commentary on the International Perspective. Journal of Adolescence Health 33: 219-225.

Miller, Linda, Emma Pollard, Fiona Neathey, Darcy Hill, und Helen Ritchie. 2005. Gender segregation in apprenticeships. Manchester: EOC.

Rauch, Angela, und Karen Schober. 1996. Geschlechtsspezifisches Rekrutierungsverhalten westdeutscher Betriebe bei der Ausbildung und Beschäftigung von Auszubildenden und Fachkräften in anerkannten Ausbildungsberufen. In Hürden im Erwerbsleben. Aspekte beruflicher Segregation nach Geschlecht. Beiträge zur Arbeitsmarkt- und Berufsforschung Nr. 198, Hrsg. Sabine Liesering und Angela Rauch, 17-45. Nürnberg: IAB.

SECO, Staatssekretariat für Wirtschaft. o.J. Wegleitung zu den Verordnungen 3 und 4 zum Arbeitsgesetz. Bern: SECO (Stand: November 2011).

SKBF, Schweizerische Koordinationsstelle für Bildungsforschung. 2010. Bildungsbericht Schweiz 2010. Aarau: SKBF.

Trappe, Heike. 2006. Berufliche Segregation im Kontext. Über einige Folgen geschlechtstypischer Berufsentscheidungen in Ost- und Westdeutschland. Kölner Zeitschrift für Soziologie und Sozialpsychologie 58(1): 50-78. 


\section{Anhang}

Tab. 1: Untersuchte Betriebe nach Art, Anzahl Mitarbeiter, betrieblicher Position des Interviewpartners, und Ausbildungsberuf 
Tab. 1: Untersuchte Betriebe nach Art, Anzahl Mitarbeiter, betrieblicher Position des Interviewpartners, und Ausbildungsberuf

\begin{tabular}{|c|c|c|c|c|}
\hline [Nr.] & Betriebsart & $\begin{array}{l}\text { Anzahl } \\
\text { Mitarbeiter }\end{array}$ & $\begin{array}{l}\text { Betriebliche Position } \\
\text { Interviewpartner }\end{array}$ & $\begin{array}{l}\text { Ausbildungsberuf } \\
\text { (offene Lehrstelle) }\end{array}$ \\
\hline 1 & Autogarage & $1-3$ & Geschäftsinhaber & Automonteur/in \\
\hline 2 & Karosseriewerk & $11-20$ & Geschäftsführer & Autolackierer/in \\
\hline 3 & Autogarage & $7-10$ & Geschäftsinhaber & Automonteur/in \\
\hline 4 & Autogarage & $4-6$ & Werkstattleiter & Anlehre Autogewerbe \\
\hline 5 & Lackiererei & $4-6$ & Geschäftsführer & Autolackierer/in \\
\hline 6 & Autogarage & $11-20$ & Filialleiter & Automonteur/-mechaniker/in \\
\hline 7 & Autogarage & $101-250$ & Ausbildner & Autolackierer/in \\
\hline 8 & Karosseriewerk & $11-20$ & Geschäftsinhaber & Autolackierer/in \\
\hline 9 & Autogarage & $101-250$ & Personalverantwortliche & Automonteur/-mechaniker/in \\
\hline 10 & Autogarage & $51-100$ & Leiter Karosserie & Autolackierer/in \\
\hline 11 & Autogarage & $11-20$ & Ausbildungsleiter & Automechaniker/in \\
\hline 12 & Karosseriewerk & $11-20$ & Vorsteher Administration & Autolackierer/in \\
\hline 13 & Karosseriewerk & $11-20$ & Betriebsleiter & Autolackierer/in \\
\hline 14 & Lackiererei & $11-20$ & Geschäftsleiter & Autolackierer/in \\
\hline 15 & Autogarage & $51-100$ & Ausbildungsleiter & Automonteur/-mechaniker/in \\
\hline 16 & Autogarage & $51-100$ & Technischer Betriebsleiter & Automechaniker/in \\
\hline 17 & Autogarage & $11-20$ & Werkstattleiter & Automechaniker/in \\
\hline 18 & Lackiererei & $4-6$ & Geschäftsinhaber & Autolackierer/in \\
\hline 19 & Autogarage & $11-20$ & Verwalter & Automonteur/-mechaniker/in \\
\hline 20 & Autogarage & $21-50$ & Technischer Betriebsleiter & Automonteur/-mechaniker/in \\
\hline 21 & Autogarage & $51-100$ & Ausbildungsleiter & Autolackierer/in \\
\hline 22 & Karosseriewerk & $4-6$ & Werkleiter & Autolackierer/in \\
\hline 23 & Autogarage & $7-10$ & Werkstattleiter & Automonteur/in \\
\hline 24 & Karosseriewerk & $21-50$ & Betriebs- u. Personalleiter & Autolackierer/in \\
\hline 25 & Karosseriewerk & $1-3$ & Geschäftsinhaber & Autolackierer/in \\
\hline 26 & Autogarage & $11-20$ & Filialleiter & Automonteur/-mechaniker/in \\
\hline 27 & Autogarage & $11-20$ & Werkstattleiter & Automechaniker/in \\
\hline
\end{tabular}

